\title{
Claroscuros en la formación de técnicos superiores universitarios. El fomento de las habilidades para la argumentación en el aula
}

Chiaroscuros of the Technical Higher Education. The Incentivizing of Argumentative Abilities in the Classroom

Claroscuros na formação de técnicos de universidades superiores. Promoção de habilidades de argumentação na sala de aula

Mónica Muñoz Muñoz

Universidad Autónoma de Zacatecas, Área Académica de Humanidades y Educación, México mmunozmunoz74@gmail.com https://orcid.org/0000-0002-3948-2815

Ivonne Ibarra Peña Universidad Autónoma de Zacatecas, Área Académica de Humanidades y Educación, México https://orcid.org/0000-0002-6168-0607 


\section{Resumen}

En el abanico de posibilidades de educación superior en México, existe un subsistema que pertenece a la Coordinación General de Universidades Tecnológicas y Politécnicas denominado universidad tecnológica, producto de las políticas educativas ligadas al sector productivo. Este trabajo es resultado de un estudio de caso sobre la realización de textos argumentativos en la Universidad Tecnológica del Norte de Aguascalientes, México; tiene como objetivo el análisis de la práctica de la argumentación escrita en los momentos en que se utiliza para la construcción del conocimiento. La metodología es de carácter mixto. Recogió datos cuantitativos y cualitativos que permitieron el abordaje del objeto de investigación de manera compleja. Durante dos meses se observó de manera pasiva el trabajo y la interacción en el aula entre alumnos y maestros; también se analizaron textos escritos por 60 estudiantes bajo la etiqueta de "argumentativos". En cualquier tipo de educación superior, es indispensable concebir la escritura como un proceso que permita clarificar, organizar y compartir ideas, además de crear interrogantes y conclusiones, para que los estudiantes se puedan integrar al mercado laboral con las habilidades cognitivas propias del discurso argumentativo. Sin embargo, en la formación de técnicos superiores universitarios no se propicia el desarrollo de la argumentación desde la práctica educativa.

Palabras clave: argumentación, aprendizaje, lenguaje escrito, trabajos de clase.

\section{Abstract}

Within the range of options for higher education in Mexico, there is a subsystem belonging to the Coordinación General de Universidades Tecnológicas y Politécnicas called Technological University, product of the educational policies linked to the productive sector. This work is the result of a case study on the production of argumentative texts in the Universidad Tecnológica del Norte de Aguascalientes, in Mexico. Its objective is to analyse the practice of written argumentation when used in the construction of knowledge. The methodology is mixed. For two months the work and interaction in the classroom between students and teachers was passively observed. Texts written by 60 students under the label of "argumentative" were also analyzed. In any type of higher education, it is essential to conceive writing as a process that allows the clarifying, organizing and sharing of ideas, as well as creating questions and conclusions, so that students can integrate into the job market 


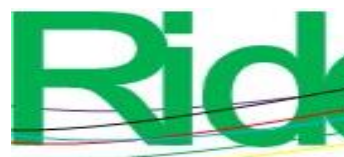

Revista Iberoamericana para la Investigación y el Desarrollo Educativo ISSN $2007-7467$

with the cognitive skills of argumentative discourse. Nonetheless, there is no encouragement towards the development of argumentation in the educational practice used to train students of technical higher education.

Keywords: argumentation, learning, written language, classwork.

\section{Resumo}

No leque de possibilidades do ensino superior no México, existe um subsistema pertencente à Coordenação Geral de Universidades Tecnológicas e Politécnicas, denominado universidade tecnológica, produto de políticas educacionais vinculadas ao setor produtivo. Este trabalho é o resultado de um estudo de caso sobre a elaboração de textos argumentativos na Universidade Tecnológica do Norte de Aguascalientes, México; seu objetivo é a análise da prática da argumentação escrita nos momentos em que é utilizada para a construção do conhecimento. A metodologia é de natureza mista. Dados quantitativos e qualitativos coletados que permitiram a abordagem do objeto de pesquisa de maneira complexa. Durante dois meses, foi observado passivamente o trabalho em sala de aula e a interação entre alunos e professores; Também foram analisados textos escritos por 60 estudantes sob o rótulo de "argumentativo". Em qualquer tipo de ensino superior, é essencial conceber a escrita como um processo que permita esclarecer, organizar e compartilhar idéias, além de criar perguntas e conclusões, para que os alunos possam se integrar ao mercado de trabalho com as habilidades cognitivas do discurso argumentativo. No entanto, no treinamento de técnicos de universidades superiores, o desenvolvimento da argumentação a partir da prática educacional não é incentivado.

Palavras-chave: argumentação, aprendizagem, linguagem escrita, trabalho de classe. 


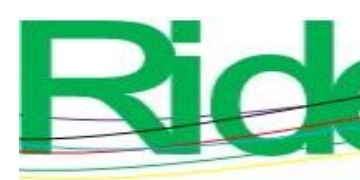

Revista Iberoamericana para la Investigación y el Desarrollo Educativo ISSN $2007-7467$

\section{Introducción}

En casi cualquier etapa de la vida escolar, pero especialmente en la universitaria, la escritura es un elemento transversal de la formación; en cualquier campo del conocimiento, es el eje articulador de lecturas, reflexiones y nuevos aprendizajes que permite al individuo concretar en palabras la transformación que de manera cognitiva experimenta. Es cierto, como argumenta Daniel Cassany (2012), que escribiendo se aprende; la escritura se puede utilizar para comprender de mejor manera cualquier tema. Sin embargo, las repercusiones de dicha competencia van más allá si se toma en cuenta que permite la progresión de los procesos de pensamiento $y$, por tanto, forja mentes capaces de enfrentarse a los retos de la vida profesional y cotidiana.

La escritura no solo permite el registro y la acumulación de conocimientos, también promueve una forma específica de aprender: una cultura de aprendizaje (Pozo, 2012, p. 92). Además, crea nuevas posibilidades cognitivas, nuevas capacidades o competencias que se concretan en el pensamiento crítico y reflexivo; aprender a escribir es aprender a pensar. El ejercicio de la escritura prepara al estudiante para el afrontamiento de retos en diversas situaciones y contextos.

En la medida en que la educación se compromete con el desarrollo del pensamiento crítico en las aulas, los estudiantes son capaces de argumentar y emitir juicios propios. Es el camino del razonamiento y la reflexión que culmina en la formación de ciudadanos democráticos, capaces de realizar procesos mentales que desemboquen en la argumentación, la cual se utiliza en los ámbitos públicos y privados para rebatir, para lograr la adhesión del interlocutor hacia formas de pensamiento y acción.

Además de la posibilidad de intervención en el punto de vista de quien nos escucha, la argumentación conlleva otros beneficios para quien la practica. La mente despierta y se flexibiliza; hay una mejoría del individuo que, a través del pensamiento crítico, otorga valor a los juicios impuestos por la vida familiar, la laboral e incluso la religiosa (Weston, 2016, p. 16).

El dominio del discurso argumentativo por parte de los estudiantes de nivel superior es un elemento fundamental del desarrollo humano y social. "Por ser un componente de la interacción humana, la competencia argumentativa, definida como la habilidad para producir argumentos, en todas las culturas es factor clave del éxito político, laboral, comunitario y familiar" (Gómez, 2012, p. 103). 


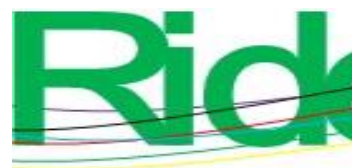

Revista Iberoamericana para la Investigación y el Desarrollo Educativo ISSN $2007-7467$

El presente artículo es el resultado de un estudio de caso sobre el manejo del discurso argumentativo de un grupo de alumnos de la Universidad Tecnológica del Norte de Aguascalientes (UTNA), que tuvo como eje el análisis de la práctica del discurso argumentativo en la cultura del aprendizaje, es decir, en los momentos en que se utiliza en la construcción del conocimiento y del desarrollo académico.

El estudio de caso se hizo a partir de tres momentos de la investigación, con un enfoque metodológico cuanti-cualitativo: 1) la observación no participante, sustentada en la metodología de investigación cualitativa; 2) la encuesta, que permitió encontrar parámetros del uso de la argumentación a través de la percepción del alumno, y c) la aplicación de un ejercicio escrito, a través del cual se evaluó la competencia argumentativa escrita.

\section{La escritura argumentativa}

La enseñanza de la lengua materna en el medio universitario no ha de conformarse con el dominio gramatical, tal postura implica la concepción de la lengua como un mero código que ha de combinarse en secuencias aceptables. Es necesario que, como asevera Álvaro Díaz (2015) en La argumentación escrita, en un texto las palabras y las oraciones pueden adquirir connotaciones diferentes de las que se les podrían asignar de forma aislada; más que la construcción de oraciones dentro de la norma, la competencia argumentativa implica la hechura de textos coherentes, que ejerzan fenómenos como la cohesión, la inferencia, la presuposición y la delimitación de intenciones (Díaz, 2015, p. 35).

En el nivel universitario se debe superar la barrera del dominio gramatical y exigir que sea el principio comunicativo el que impere. "La enseñanza del lenguaje debe contribuir a la adquisición y al dominio de las destrezas comunicativas más habituales en la vida de las personas: hablar, escuchar, leer, entender y escribir" (Lomas, 2017, p. 49).

De manera concreta, además de considerar reglas sintácticas en la producción de un texto, el estudiante universitario debe contar con competencias indispensables como poder diseñar el plan de un texto explicativo y utilizar estrategias que garanticen coherencia, cohesión y pertinencia, características propias de los textos argumentativos.

En Occidente, la argumentación se encuentra dentro de la tradición retórica, la disciplina considerada como la actividad política por excelencia: 
La forma de convivencia humana básica es lingüística y, por ende, la habilidad de manejar críticamente el lenguaje es fundamental en sociedades como la nuestra.

Bajo esta perspectiva la Retórica no es simplemente una disciplina secundaria de la lingüística; mucho menos puede considerarse como sinónimo de discurso vacío o manipulador. De igual manera, consideramos importante hacer una distinción entre la Retórica como ciencia y arte de la argumentación y la retórica (con minúscula) como la habilidad o capacidad de deliberar sobre lo que es adecuado en una situación, es decir, la habilidad para argumentar (Monzón, 2011, p. 42).

Según Peña (2008, p. 4), los problemas en la construcción de textos argumentativos por parte de los alumnos estriban en la exposición de ideas de forma aislada y esquemática, en la falta de un plan previo para la construcción del texto y en el desconocimiento de las convenciones de la escritura académica.

Según los resultados que aquí presentamos, la escritura en las universidades tecnológicas cumple primordialmente una función evaluativa. Cuando no es así, el acto de escribir se convierte en una actividad mecánica a través del dictado, del copiado de textos escritos en pizarrón o de la contestación de un cuestionario que requiere respuestas textuales. Se trata de acciones vinculadas a una reproducción precisa de los textos originales y, desafortunadamente, alejadas del desarrollo de habilidades cognitivas.

Es necesario insistir en la concepción de la escritura como un proceso de comunicación capaz de clarificar, organizar y compartir ideas de forma explícita, además de crear y compartir interrogantes sobre el contenido del aprendizaje. Las acciones anteriores provocan la reflexión en el momento de escribir y, por tanto, la construcción del conocimiento en el estudiante porque implican la utilización de la palabra escrita en el afianzamiento y desarrollo de las ideas, teniendo la posibilidad de volver a estas para clarificarlas y, en su momento, reafirmarlas o refutarlas.

En nivel universitario, sea cual fuere el campo de acción del futuro profesionista, “aprender a escribir es a la vez aprender a pensar crítica y creativamente. Dicho de otro modo, desarrollamos mejor nuestra capacidad para pensar críticamente a medida que aprendemos a escribir textos académicos (ensayos, informes, reseñas, monografías)” (Díaz, 2015, p. 32). 


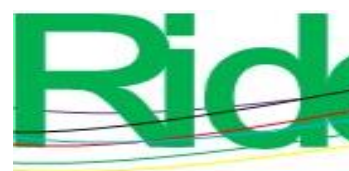

El discurso en sus diferentes formas (exposición, descripción, narración y argumentación) tiene sendos propósitos. La argumentación, tema fundamental de este artículo, es un elemento comunicativo indispensable para que los universitarios realicen razonamientos propios del pensamiento crítico.

Los textos argumentativos son escritos que permiten crear conexiones con otras opiniones e integrar conocimientos de diferentes fuentes de información hasta forjar el propio conocimiento. En otras palabras, "la argumentación es una forma de convencer o de lograr una adhesión de un determinado auditorio, apoyándose en criterios racionales” (Díaz, 2015, p. 5).

Argumentar implica hacer comparaciones a través del hallazgo de semejanzas o diferencias; realizar analogías; definir términos, conceptos e ideas; analizar causas y efectos. Los párrafos de la escritura argumentativa tienen propósitos especiales que permiten ordenar el texto en su conjunto, frecuentemente bajo el esquema de una introducción seguida por el desarrollo de la idea central con sus respectivas premisas y un apartado conclusivo.

$\mathrm{Si}$, como se dijo anteriormente, las formas del discurso interactúan entre sí, es posible que en la argumentación se utilicen narraciones o descripciones como argumentos. También es posible que una sola oración compleja mantenga la estructura de introducción, hipótesis, argumentación y conclusión, que a su vez pertenecerá a una estructura argumentativa mayor caracterizada por un mayor nivel de profundidad pero hecha con el mismo procedimiento y estructura; el texto argumentativo está construido a su vez por mini estructuras argumentativas.

El pensamiento crítico es el telón de fondo del texto argumentativo, por lo cual este se convierte en un discurso que vincula a las humanidades con el resto de las áreas del conocimiento y en una herramienta necesaria de cualquier profesionista para comunicar, persuadir o refutar.

Además, la escritura argumentativa, al incluir la coherencia en el planteamiento de las ideas, supone una organización o disposición de párrafos y oraciones con un fin comunicativo determinado, donde todo argumento puede ser modificable, se puede replantear y cuestionar debido a que sirve de base para posteriores argumentaciones tanto del autor como de sus lectores. 


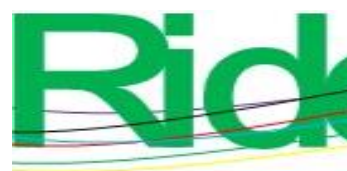

2

de
Revista Iberoamericana para la Investigación y el Desarrollo Educativo ISSN $2007-7467$

A nivel universitario, la elaboración de textos de carácter argumentativo es fundamental, como lo confirma Cristián Santibáñez (2018), quien en Origen y función de la argumentación explica de manera amplia una de sus principales tesis: en contextos académicos en los que se preserva, genera y difunde conocimiento a través de documentos escritos, la argumentación es una condición intrínseca del discurso que le aporta solidez al escrito y prestigio al autor.

\section{La universidad tecnológica y la escritura}

En el abanico de posibilidades de educación superior en México, existe un subsistema que pertenece a la Coordinación General de Universidades Tecnológicas y Politécnicas [CGUT] (2016) denominado universidad tecnológica, que es producto de las políticas educativas ligadas al sector productivo y que tiene como misión formar técnicos superiores universitarios. La UTNA, que pertenece al subsistema de universidades tecnológicas, brinda una formación técnica bajo el manejo de la ciencia y la tecnología

La UTNA inició ofertando cursos de dos años a estudiantes egresados de la educación media superior con el fin de otorgar el título de técnico superior universitario (TSU) y de incorporar a sus egresados en un corto plazo al campo productivo. El currículo incluye $30 \%$ de teoría y $70 \%$ de conocimiento aplicado o práctico que incluye visitas a empresas y proyectos (CGUT, 2016).

Al analizar los documentos base de las universidades tecnológicas como los son el plan de estudios, el perfil profesional y la matriz de competencias del profesionista en relación con el fomento y desarrollo de la escritura, se constató que la competencia escrita se define como "genérica", por lo que debe estar presente en la totalidad del trayecto formativo del estudiante, pero especialmente en el momento de evaluar el progreso del alumno en cada materia.

Así, la escritura es una actividad de corte evaluativo. En cada asignatura se realizan diferentes tipos de textos, aunque predominando como forma discursiva la descripción porque los trabajos académicos, en su mayoría, son reportes, bitácoras, manuales, informes, inventarios, planes maestros, listas de verificación, procedimientos y listas de verificación. 


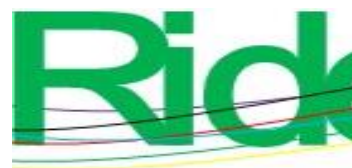

Revista Iberoamericana para la

Investigación y el Desarrollo Educativo

ISSN $2007-7467$

Además, en el plan de estudios de las diferentes carreras de la UTNA se incluye la unidad didáctica Expresión Oral y Escrita, la cual, por sus contenidos y características, es la responsable de promover la competencia linguiística en sus diferentes modalidades y formas discursivas. Sin embargo, dado que la escritura es una competencia genérica, la argumentación debe ser promovida también por el resto de las unidades didácticas o materias. Para justificar tal propuesta un argumento de peso puede ser el siguiente:

Otro riesgo de enseñar a escribir y leer en espacios curriculares ad hoc es la potencial irrelevancia o falta de sentido que los alumnos puedan encontrar en ellos (Myers Zawacki y Taliaferro Williams, 2001). Los estudiantes que han elegido como carrera la Sociología o la Física, por ejemplo, suelen preguntarse por qué deben cursar un taller general de escritura y/o lectura. En cambio, si el profesor de Sociología dedicara tiempo de clase a discutir el texto que da para leer y a revisar colectivamente parte de lo que ellos han escrito, probablemente se sentirían guiados en lo que eligieron formarse. Igualmente, si en el taller de comunicación — a cargo de un docente de Física y un profesor de escritura - se acordara y orientara un proyecto de escritura para participar en un congreso de la especialidad, los estudiantes se sentirían convocados. (Carlino, 2013, párr. 28).

En la medida en que los docentes utilicen la escritura como instrumento del aprendizaje más que como la oportunidad de evaluación, los jóvenes podrán dejar de sentirse amenazados por las palabras que ponen por escrito, por los textos que tímidamente escriben, así como por aquellos que realizan mecánicamente donde han de repetir los textos fuente; implícitamente han aprendido que no necesitan demostrar una postura y argumentarla.

Respecto a la importancia de las habilidades argumentativas sabemos lo siguiente:

La argumentación no se puede asociar ni dependerá solo de un texto escrito, pues más que un tipo de texto o discurso, la argumentación es una actitud frente a la ciencia, una forma de mirar la producción de conocimiento científico y de comunicarlo, una manera de hacer bien para influir y persuadir desde lo que decimos, pero también desde lo que hacemos y somos (Zambrano, Orozco y Caro, 2016, p. 53). 


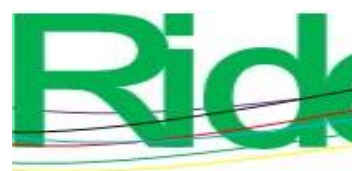

Revista Iberoamericana para la Investigación y el Desarrollo Educativo ISSN $2007-7467$

Por lo anterior, no puede dejarse de lado el fomento y la posibilidad de desarrollo de las habilidades argumentativas, independientemente de que el perfil educativo sea tecnológico o no, independientemente del nivel escolar, sea universitario o no.

\section{Metodología}

Este artículo es producto del estudio de caso realizado en la UTNA durante el ciclo escolar 2017-2018 con los alumnos del cuarto semestre de la carrera de Mantenimiento Industrial, una de las carreras con mayor antigüedad y demanda dentro de este subsistema de universidades tecnológicas.

La metodología del estudio de caso está basada en un enfoque de investigación mixto, es decir, tiene una fase de recolección de datos cuantitativos y otra basada en la observación no participante que implica la recopilación de datos cualitativos. Las investigaciones humanísticas y educativas permiten el establecimiento de relaciones entre los métodos cuantitativos y cualitativos; los primeros implican la acumulación y la lectura estadística de datos, mientras que los segundos son pertinentes para analizar las particularidades del objeto a investigar.

Como explica Javier Núñez (2017, p. 245), ambos métodos de investigación pueden contraponerse o complementarse. En esta última postura se encuentra el método mixto, que se rige bajo el principio de retroalimentación entre observaciones cuantitativas y cualitativas para lograr una mayor comprensión del objeto investigado, es decir, un acercamiento complejo, enriquecido de acuerdo con la propuesta de Edgar Morin (2008).

Además, en la investigación educativa, se recomienda el enfoque mixto porque permite registrar el punto de vista de los participantes, en este caso, los estudiantes de la UTNA, cuya mirada y posición alcanza a ser parte de la investigación al incluir también la actuación de los docentes y la descripción del clima escolar.

Es viable el planteamiento de un diseño de método mixto para la aproximación a temáticas de estudio en el ámbito pedagógico, en especial, cuando hay una evidente intención del investigador o investigadora para otorgar voz a los participantes y a las participantes; en ese sentido, cuando no solo se desea la obtención de datos numéricos, sino también se busca la visión más íntima del participante, los datos cualitativos cobran un papel relevante (Pereira, 2011, p. 27). 


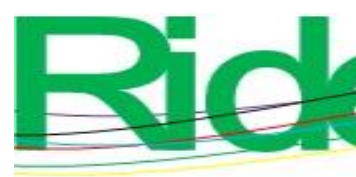

\section{Revista Iberoamericana para la Investigación y el Desarrollo Educativo ISSN $2007-7467$}

El estudio de caso tiene como una de sus virtudes el acercamiento directo con el centro de toda institución educativa, los alumnos, que son quienes experimentan directamente los momentos en que la institución y los maestros promueven la escritura. El enfoque mixto, por tanto, se justifica para cumplir los objetivos de la investigación.

Los informantes, 60 estudiantes de la carrera de Mantenimiento Industrial de la UTNA, en el momento de la obtención de los datos tenían entre 18 y 20 años de edad. Aunque cursaban sus estudios universitarios en Aguascalientes, eran originarios de municipios pertenecientes al estado de Zacatecas (Cd. Cuauhtémoc, Luis Moya, Loreto, Luis Moya y Villa García) y de otros del mismo estado hidrocálido (Cosío, Pabellón de Arteaga, Rincón de Romos, Asientos, San Francisco y Tepezalá). A pesar de poder pertenecer a entidades políticas distintas, los informantes compartían rasgos culturales determinados por el contexto rural, aunque poseían un interés genuino por el avance técnico promovido por la universidad tecnológica.

Tomando como base la propuesta de metodología mixta de Núñez (2017, p. 644), las fases metodológicas fueron cuatro y se describen a continuación.

\section{Fase uno. Cuantitativa}

Durante el mes de septiembre de 2017, se obtuvieron los datos a través de la aplicación a los 60 informantes de un ejercicio escrito basado en la propuesta de Fonseca (2011, p. 296). Los estudiantes debían realizar un texto con una estructura básica de argumentación: una premisa sostenida y desarrollada a través de argumentos hasta llegar a una conclusión. Fue fundamental que los textos se construyeran con cohesión y coherencia, sin embargo, en su evaluación también se tomaron en cuenta elementos formales como el tipo de nexos utilizados y la precisión léxica.

\section{Fase dos. Modalidad de desarrollo}

Se analizaron estadísticamente los datos obtenidos en la fase uno, a partir de los cuales se elaboró un cuestionario que permitió la obtención de datos cualitativos y cuantitativos. En esta etapa el objetivo principal fue indagar el ejercicio y la percepción del alumno respecto a su desempeño académico a través de la escritura, es decir, saber cómo conciben la escritura los estudiantes y qué importancia le dan en la construcción del conocimiento a la argumentación. Las preguntas fueron las siguientes: 


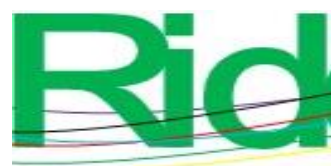

Revista Iberoamericana para la Investigación y el Desarrollo Educativo ISSN $2007-7467$

1) ¿Cuáles son los trabajos que realiza con mayor frecuencia como estudiante de la UTNA, tales como el informe, el ensayo, el resumen, la síntesis, el cuestionario u otro? Enúncielos con base en la frecuencia de uso.

2) Al momento de elaborar un trabajo escrito, ¿sigue algún procedimiento? Si es así, descríbalo.

3) ¿Cuáles son sus puntos fuertes y débiles en la escritura?

4) ¿Le gusta escribir? ¿Por qué?

5) ¿Para qué es más importante escribir? ¿Para realizar actividades laborales, académicas, interpersonales o profesionales?

6) ¿Considera importante que un TSU tenga una buena competencia escrita?

7) ¿Qué elementos de la escritura argumentativa considera necesarios para poder desempeñar su profesión?

8) ¿Considera que la UTNA ha contribuido a mejorar su escritura?

\section{Fase tres. Cualitativa}

A través de la observación no participante se estuvo en contacto con el objeto de la investigación de manera multidimensional, ya que la investigación in situ permitió observar contextos, reacciones, interacción maestro-alumno y situaciones diversas. Durante los meses de octubre y noviembre de 2017 se observaron las actividades en el aula de los alumnos y maestros de cuarto semestre de la carrera de Mantenimiento Industrial de la UTNA. En total, se observaron 26 sesiones de 50 minutos cada una, pertenecientes a las nueve asignaturas que forman parte del plan de estudios. El proceso de observación implicó registrar, con ayuda de una bitácora, las actitudes, los momentos, las finalidades y los contextos en que los alumnos utilizaron la escritura, poniendo especial detalle en la escritura argumentativa, para señalar aquello que, de manera voluntaria o involuntaria, visible u oculta, repercute en el ejercicio de la escritura por parte de los alumnos. 
Fase cuatro. Modalidad de complementariedad

Se confrontaron los resultados obtenidos en las fases precedentes, entendiendo los resultados de las fases cuantitativa y cualitativa como dos tipos de datos de distinta naturaleza que reflejan, desde perspectivas opuestas y complementarias, el mismo objeto de estudio.

\section{Resultados y discusión}

En la fase uno, de manera cuantitativa la elaboración de un texto argumentativo tuvo resultados negativos. De los 60 estudiantes que conformaron la muestra, solo $11.7 \%$ elaboró un texto argumentativo con la estructura básica: premisa o hipótesis, argumento y conclusión, es decir, apenas siete informantes. El resto de los textos producidos tuvo una estructura mucho más cercana a la descripción: se nombraron acciones y atributos de un tema que se eligió libremente; en el mejor de los casos, se dieron opiniones que no llegaron sostenerse con argumentos.

Los resultados anteriores hicieron difícil la profundización en el análisis del discurso argumentativo. La tabla 1 presenta características generales de los textos producidos por los alumnos de la UTNA. Se realizó con base en los textos argumentativos que produjeron en la primera etapa de la investigación. 
Tabla 1. Análisis cualitativo de textos argumentativos construidos por alumnos de la

UTNA

\begin{tabular}{|c|c|c|c|}
\hline Estructura & Coherencia & Cohesión & $\begin{array}{l}\text { Elementos } \\
\text { gramaticales }\end{array}$ \\
\hline $\begin{array}{l}\text { Las oraciones introductorias } \\
\text { son muy extensas, se construyen } \\
\text { con adjetivos como importante e } \\
\text { interesante sin llegar a plantear } \\
\text { una premisa. } \\
\text { Se registran oraciones cuya } \\
\text { relación con el tema no se explica. } \\
\text { Se utilizan afirmaciones como } \\
\text { argumentos. }\end{array}$ & $\begin{array}{l}\text { Se registran } \\
\text { contradicciones } \\
\text { en las ideas que } \\
\text { constituyen un } \\
\text { párrafo. } \\
\text { Predominan las } \\
\text { oraciones con } \\
\text { un solo núcleo } \\
\text { verbal, lo que } \\
\text { dificulta la } \\
\text { conexión de y el } \\
\text { desarrollo ideas. } \\
\text { Se detectan } \\
\text { vocablos } \\
\text { imprecisos } \\
\text { semánticamente. } \\
\text { No existe ni una } \\
\text { organización } \\
\text { deductiva ni } \\
\text { inductiva en la } \\
\text { ideas. }\end{array}$ & $\begin{array}{l}\text { Se utilizan } \\
\text { conectores } \\
\text { causales } \\
\text { característicos } \\
\text { de la } \\
\text { argumentación } \\
\text { tales como } \\
\text { porque, pues, } \\
\text { puesto que. } \\
\text { No se registran } \\
\text { dado que, ya } \\
\text { que, por el } \\
\text { hecho de que, } \\
\text { en virtud de } \\
\text { que. }\end{array}$ & $\begin{array}{l}\text { Se registraron } \\
\text { errores básicos } \\
\text { de puntuación y } \\
\text { ortografía, de } \\
\text { manera } \\
\text { específica en el } \\
\text { uso de las letras } \\
z, s, c ; b \text { y } v . \\
\text { No se dominan } \\
\text { las reglas de } \\
\text { acentuación. } \\
\text { Se utilizan } \\
\text { coloquialismos. }\end{array}$ \\
\hline
\end{tabular}




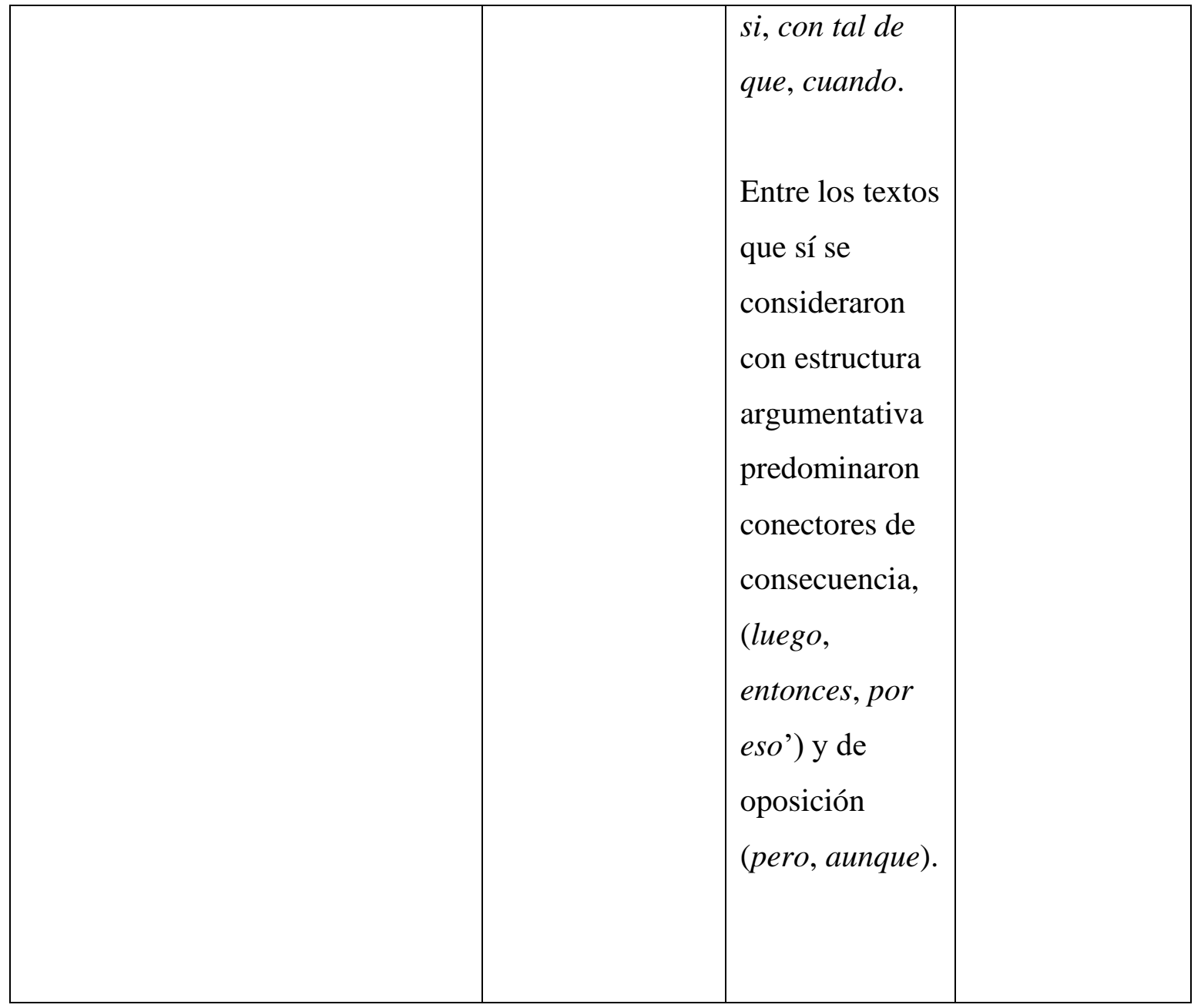

Fuente: Elaboración propia

Como lo refleja la tabla 1, las estructuras lingüísticas presentes en las opiniones argumentativas hechas por los estudiantes no son párrafos completos; en su mayoría los informantes elaboraron una o dos oraciones en las que frecuentemente repitieron una misma idea, además de limitarse a enlistar o describir en lugar de argumentar. No realizaron aseveraciones concretas y hubo una importante producción de oraciones desviadas totalmente del tema.

Cuando algunos alumnos redactaron un párrafo completo, se revisó la coherencia en sus planteamientos, así se encontraron contradicciones en las diferentes oraciones elaboradas. Además, las oraciones no alcanzaron a estar ligadas por el contenido semántico, solo el gramatical, pues se transcurría de un tema a otro de manera indiscriminada. También se produjeron imprecisiones semánticas en el nivel léxico. 


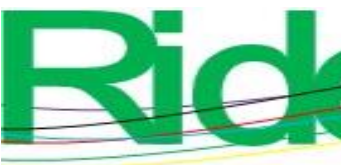

Revista Iberoamericana para la

Investigación y el Desarrollo Educativo

ISSN $2007-7467$

En lo referente a la cohesión de las oraciones, se usaron nexos inapropiados para el contexto lingüístico y una presencia significativa de muletillas, que aparentaban ser conjunciones como porque, pero, $y$, innecesarias gramaticalmente y ajenas al contexto lingüístico

En la fase dos, respecto al tipo de trabajo más recurrente donde se utiliza la escritura, los jóvenes respondieron lo que puede apreciarse en la figura 1.

Figura 1. Trabajos escritos de alumnos de TSU

\section{Trabajos que realizan los estudiantes de TSU en MI con mayor frecuencia dentro de su formación académica}

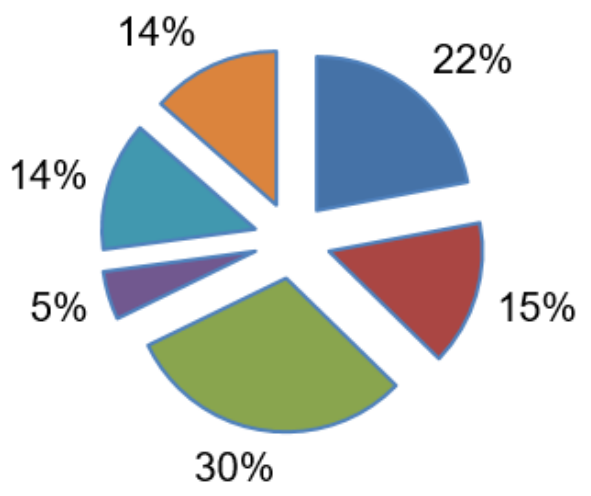

Fuente: Elaboración propia
Informes

Ensayos

Resumen

Síntesis

Cuestionarios

Otros (reporte de prácticas)

Como puede verse, según la opinión de los estudiantes, el resumen constituye una actividad cotidiana en la UTNA, obtuvo $30 \%$ de menciones, fue seguido por el informe con $22 \%$; con $15 \%$ se registró el ensayo y con 14 \% el cuestionario; en último lugar apareció la síntesis, con $5 \%$. Si este resultado se contrasta con lo observado en la fase tres, la observación no participante, puede concluirse que los alumnos han sido benévolos al juzgar los momentos en que juzgaron su escritura, pues in situ se comprobó que la frecuencia de trabajos académicos sigue el siguiente orden: resumen, informe y cuestionario; el ensayo simplemente no se ejecutó a pesar de que en la figura anterior aparece en tercer lugar. Es indudable que hay un predominio de actividades enunciativas dentro de la formación de TSU. Y con los mismos datos se corrobora que el pensamiento crítico no se atiende, en la medida en que la argumentación no es promovida. 


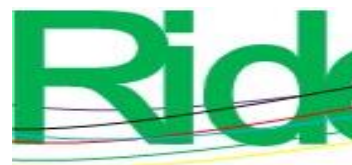

\section{Revista Iberoamericana para la Investigación y el Desarrollo Educativo ISSN $2007-7467$}

El ensayo constituye un escrito personal donde se argumenta el punto de vista, la opinión o la posición ante un tema determinado. Anthony Weston (2016, p. 92) considera que el poder realizar un ensayo argumentativo es una prueba contundente del dominio de tal discurso. Sin embargo, como se ha visto, no es un trabajo prioritario en la UTNA, a pesar de que se trata de un nivel universitario.

Respecto al reactivo "Al momento de elaborar un trabajo escrito, ¿sigue algún procedimiento? Si es así, descríbalo”, las respuestas se muestran gráficamente en la figura 2.

Figura 2. Pasos seguidos en la realización de trabajos escritos

\section{Procedimiento para realizar trabajos escritos}

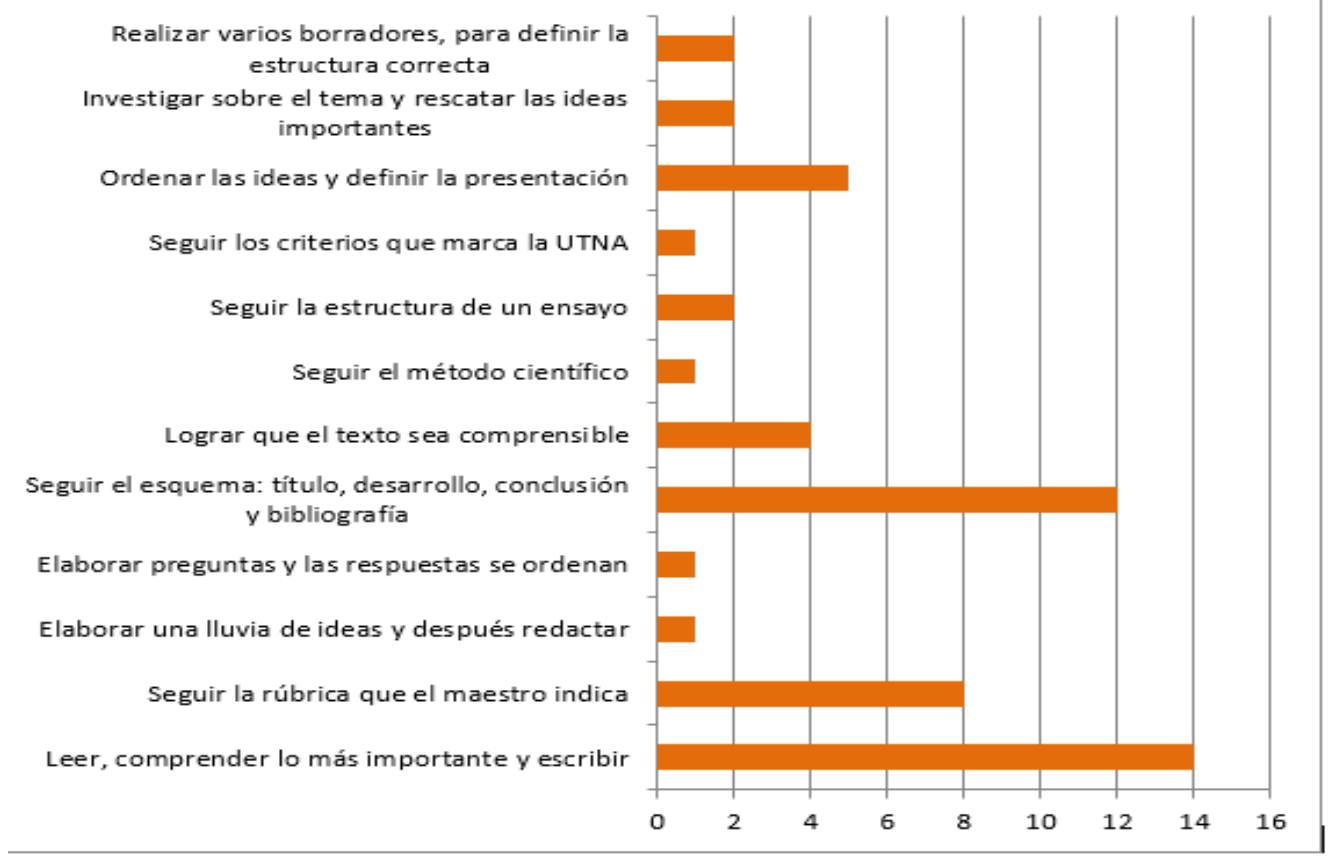

Fuente: Elaboración propia

En la figura 2 es posible advertir que de los 60 informantes que participaron en esta investigación, sólo 43 admiten seguir un procedimiento en el momento de la elaboración de trabajos escritos; el 70\%. La figura 2 también demuestra que únicamente 12 de los 60 estudiantes siguen un procedimiento similar a la estructura de los textos argumentativos: eligen un título, desarrollan ideas y llegan a conclusiones, falta - sin embargo- que tengan claro que deben redactar una hipótesis aun cuando la misma esté implícita en el título. La relativa mayoría (14) se limita a leer, comprender lo más importante y escribir. 


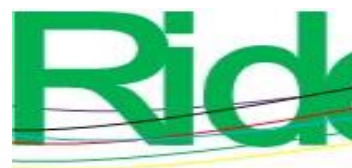

\section{Revista Iberoamericana para la Investigación y el Desarrollo Educativo ISSN $2007-7467$}

En la fase tres, de carácter cualitativo, la observación no participante permitió explicar algunos de los datos obtenidos en el primer paso metodológico de esta investigación. El trabajo de los docentes consiste, de manera predominante, en transmitir información que los alumnos copian del pizarrón a la libreta. Cuando hay algún juicio crítico por parte del docente los alumnos no retroalimentan la comunicación. Como forma de evaluación se suele revisar la libreta en la que el estudiante ha capturado lo que el maestro indicó. Como tarea, predominan los resúmenes de lecturas. En la planeación de sus trabajos, solo dos informantes dicen seguir la estructura de un ensayo argumentativo.

Todo lo anterior determina que la presencia del ensayo - considerada como poco recurrente en los estudiantes - en las diversas asignaturas no es suficiente para desarrollar en los estudiantes una argumentación o, en su defecto, elaborar una opinión, debido a que los alumnos en su mayoría solo producen oraciones aisladas carentes de elementos fundamentales como la cohesión y la coherencia textual.

A partir del estudio de caso en la UTNA se identificó que los estudiantes utilizan la escritura de forma mecánica, transcriben conceptos, esquemas, toman dictado y resuelven cuestionarios. Al final de una sesión es común que el profesor solicite los escritos de los alumnos para firmarlos, sin embargo, no hay retroalimentación o revisión; el profesor solo brinda una rúbrica.

Según los resultados de esta investigación, la escritura como proceso de construcción del conocimiento no está presente durante el desarrollo de las clases; parece solo es una ejecución mediática para registrar datos e información brindada por el docente.

Respecto al resto de los trabajos académicos como reportes de prácticas, exposiciones, reportajes, proyectos, tareas, cuentos, mapas mentales, diálogos, entrevistas y otras actividades didácticas, es de importancia la mención que hacen los estudiantes hacia la exposición como una forma de presentar los contenidos con ayuda de la forma discursiva de la argumentación, a pesar de que en este caso predomine el código oral.

Los ensayos y las exposiciones, al requerir elementos argumentativos, son actividades que se realizan con poca frecuencia en la UTNA. Algo similar sucede con la síntesis, que es la que ocupó el lugar más bajo según los resultados que se presentaron en la figura 1. La dificultad de hacer una síntesis por parte de los alumnos está relacionada con su poco ejercicio en la elaboración de opiniones argumentadas. 


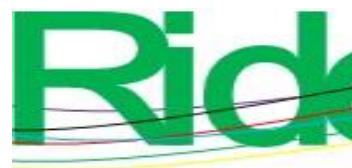

Revista Iberoamericana para la

Investigación y el Desarrollo Educativo

ISSN $2007-7467$

Respecto a la elaboración de sus trabajos escritos, los estudiantes mantienen dos posturas: hay quienes señalan que es un proceso reflexivo y hay quienes dicen que solo siguen las instrucciones del maestro sin apropiarse de lo que están escribiendo.

Dentro de la primera postura se consideraron respuestas como:

- $\quad$ Realizo varios borradores para definir la estructura correcta.

- Investigo sobre el tema y rescato las ideas importantes.

- $\quad$ Ordeno las ideas y defino la presentación.

- $\quad$ Hago que el texto sea comprensible.

- $\quad$ Elaboro preguntas o lluvia de ideas.

- $\quad$ Leo, comprendo y luego escribo.

En la segunda postura hay respuestas como:

- $\quad$ Sigo la rúbrica que el maestro indica.

- $\quad$ Sigo el procedimiento que marca la UTNA.

- $\quad$ Sigo la estructura de un ensayo.

- $\quad$ Sigo con un esquema de presentación.

Como puede verse, hay quienes tienen el propósito de utilizar la escritura para comprender y comunicar el contenido de aprendizaje y quienes dicen limitarse a seguir un esquema o plan de trabajo, dependiendo de los elementos definidos con anterioridad por el docente o la institución.

Dado que, como lo muestran nuestros resultados, la escritura no es concebida como un procedimiento, el tiempo destinado a ella es muy limitado; los alumnos durante sus clases no toman apuntes, a menos que sea por indicación del docente y los trabajos que presentan como parte de las exposiciones solo son transcripciones del contenido.

Como parte de la fase cuatro de la investigación, que reitera la complementariedad de datos cuantitativos y cualitativos, se puede afirmar que el trabajo de escritura en los alumnos es escaso, porque durante la clase el profesor es el protagonista, es él quien recurre a la técnica expositiva utilizando regularmente recursos como la presentación de diapositivas. En este contexto, los alumnos se remiten a copiar conceptos que el maestro dispone en el pintarrón, en el transcurso de la clase continúa el proceso de copiado y en algunos casos se intercala con el dictado, de manera que no hacen de la argumentación un hábito. 


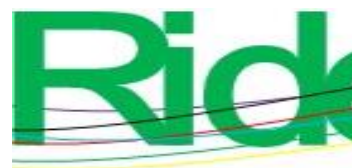

\section{Revista Iberoamericana para la Investigación y el Desarrollo Educativo ISSN $2007-7467$}

Como dijera Álvaro Díaz (2015), para poca fortuna de los países latinoamericanos, los sistemas educativos no incluyen en el currículo ni el desarrollo de todas las asignaturas suficientes actividades en las que el alumno tenga que leer críticamente y escribir con mucha frecuencia textos académicos —especialmente ensayos - sobre diversos temas. En algunos casos, porque el profesor no ha recibido un entrenamiento apropiado para realizar esta actividad, y en el peor de los casos, porque no ha comprendido la importancia de la escritura para el desarrollo de operaciones del pensamiento crítico.

Según López (2018), el pensamiento crítico es una habilidad consciente, sistemática y deliberada, producto del análisis y la valoración. Aunque todo estudiante realiza y emite juicios, la formación escolar para el pensamiento crítico le hará valerse de información suficiente, sopesando los argumentos y desentrañando las premisas que los sostienen.

Sin embargo, los datos que esta investigación recopiló demuestran que lejos de analizar o construir una argumentación y, por ende, desarrollar el pensamiento crítico, los estudiantes de la UTNA se limitan a escuchar al maestro y esperan su indicación para tomar apuntes, alejando el proceso de escritura de la construcción del propio discurso argumentativo.

A partir de los datos presentados, es válido decir que la escritura cumple una función evaluativa, se utiliza para obtener una calificación a través del cumplimiento de tareas, de la contestación de exámenes o del registro mecánico de apuntes dirigidos por el profesor. Se escribe para entregar un producto que permita la obtención de una firma sin atender una planificación del texto o una evaluación del contenido retórico.

En el artículo de Bañales, Vega, Araujo, Reyna y Rodríguez (2015) se propone que la argumentación escrita tiene una diversidad de formas, por lo que en los contextos universitarios las intervenciones educativas deben ser acordes con las necesidades de formación en los géneros textuales, las tareas y competencias argumentativas de disciplinas específicas.

Sin embargo, los datos recopilados demuestran que, en la medida en que no se exige o se fomenta la escritura de ensayos o de textos argumentativos, el único género textual que se promueve es la descripción a través de informes escuetos y reportes de prácticas. 


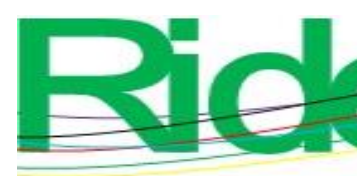

Revista Iberoamericana para la

Investigación y el Desarrollo Educativo

ISSN $2007-7467$

\section{Conclusiones}

De manera errónea, la escritura puede ser considerada como un código adquirido en la primaria que en los sucesivo servirá para la expresión y comunicación del individuo durante la vida cotidiana. Sin embargo, es necesario que sea vista más allá, como vía para la apropiación de la cultura, como elemento indispensable en la construcción del conocimiento y como herramienta insustituible en el desarrollo, la transformación y la valoración del aprendizaje. Antes de realizar textos argumentativos, el aprendiz debe desarrollar el pensamiento crítico a través del análisis, la comparación y la jerarquización, entre otras habilidades de dicho pensamiento.

El enfoque metodológico mixto bajo el que esta investigación se llevó a cabo permitió recoger datos concretos. Solo $11.7 \%$ de los estudiantes fue capaz de elaborar un texto argumentativo con una estructura básica. Según la percepción de los alumnos, el ensayo ocupa el tercer lugar en la frecuencia de elaboración de trabajos académicos (el primero lo tiene el resumen, con 30 \%; el segundo, los informes con 22 \%). En la obtención de datos cualitativos se comprobó la poca presencia de cualquier trabajo relacionado con la escritura, por ello, es entendible que el ensayo tenga apenas $15 \%$ de hechura, según la percepción estudiantil.

En el contexto de la UTNA se ha corroborado que el desarrollo de la escritura argumentativa en estudiantes de TSU está determinado por las características del propio subsistema, es decir, por el acuerdo implícito de que los alumnos se convertirán en técnicos industriales, para lo cual no es necesario que hayan desarrollado el pensamiento crítico.

La UTNA es reconocida como una institución que se caracteriza por brindar una educación que tiende al uso y dominio de la tecnología. Además, pertenece a un subsistema dirigido hacia la población rural o de zonas urbanas marginadas. El servicio educativo que brinda la UTNA tiene ventajas como el acceso de sus egresados a un trabajo catalogado de mando medio dentro de la industria. Se ha convertido en una opción viable para una población que anteriormente no tenía la posibilidad de estudiar una profesión.

Por ello, no es posible, en el contexto de la educación superior, considerar como criterio único el capacitar a un estudiante para la ejecución de un trabajo; la meta de la educación, independientemente del nivel educativo, debe ser el ejercicio del pensamiento crítico unido al pensamiento creativo, solo así podrá alcanzarse la emancipación 


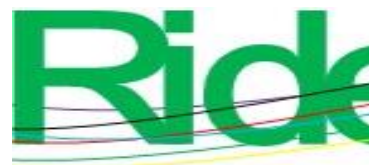

Revista Iberoamericana para la Investigación y el Desarrollo Educativo ISSN $2007-7467$

comunicativa, también llamada desalienación expresiva y comprensiva, con la que es posible crear aulas y, por ende, sociedades democráticas.

Debido a las características de sus estudiantes, la UTNA debe realizar una adaptación curricular donde el modelo educativo contemple las necesidades y las expectativas académicas de los jóvenes. Los documentos que rigen institucionalmente a la UTNA plantean la disposición de los aprendizajes de manera práctica, sin embargo, en los docentes, al impartir clases, predomina la exposición teórica de contenidos, lo que da como resultado que los estudiantes recurran a la memorización y dejen de lado el fortalecimiento de las competencias lingüística, argumentativa y cognitiva.

Respecto al desarrollo de la argumentación como producto de trabajos escritos, se concluye que a pesar de que en la UTNA la escritura se describe como una competencia genérica, como una actividad transversal, solo se ejecuta como producto de una orden para evaluar el aprendizaje de los contenidos en las diferentes asignaturas.

Aunque la escritura en los alumnos debería implicar mayor número de horas prácticas, se detectó una preferencia desbalanceada por los saberes teóricos de cualquier materia. Se escribe para elaborar trabajos de tipo descriptivo como el informe, el reporte, el inventario, el manual y el plan maestro.

La escritura argumentativa se aborda solo desde la materia de Expresión Oral y Escrita, la cual es interpretada como la responsable de promover la elaboración de textos con una estructura gramatical correcta, pero no de promover la hechura de textos que se utilizarán para apoyar o refutar un punto de vista concreto, y cumplir con ello objetivos comunicativos y autorrealizadores.

A partir de lo anterior se puede aseverar que la escritura está planteada como un rasgo necesario pero no fundamental para el aprendizaje de los estudiantes de TSU. De manera oficial se pretende que dicha habilidad lingüística sea una competencia transversal, sin embargo, se corroboró que no se dedica atención al acto de escribir. Los alumnos no realizan apuntes o textos de diferentes formas discursivas, solo transcriben o escuchan la exposición del docente.

Los maestros utilizan la escritura para evaluar el contenido de aprendizaje, pero no para el desarrollo de este, es decir, no se llevan a cabo acompañamientos o asesorías en el proceso de escribir que repercutan en los procesos cognitivos. 


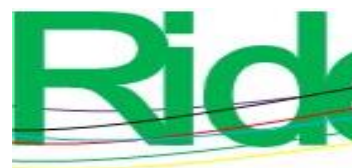

\section{Revista Iberoamericana para la Investigación y el Desarrollo Educativo ISSN $2007-7467$}

Por lo anterior es pertinente señalar que, en el contexto de la clase de lengua materna en el nivel universitario, además de la supervisión del texto final que incluya el dominio gramatical reflejado en aspectos como la ortografía, la sintaxis y la puntuación, se ahonde en la precisión léxica, en los aspectos retóricos del texto y, como se decía en las primeras páginas de este artículo, en la coherencia y la cohesión.

En lo que toca a las formas discursivas, los resultados de esta investigación demuestran que en la UTNA predominan las actividades enunciativas, después las descriptivas y con menos rango de aparición están las argumentativas.

Muchas veces la escritura se ve reducida a términos ortográficos y, en el mejor de los panoramas, sintácticos. Sin embargo, si se le entiende desde los niveles funcional y comunicativo sus alcances son mucho mayores. Ya no se puede pretender aprender a escribir en la universidad bajo un método memorístico, tradicional, que resta significado al proceso cognitivo.

La falta de un proceso reflexivo y crítico en el desarrollo de la escritura impide que la educación superior de México se convierta a su vez en la formación de mentes críticas capaces de desarrollarse en cualquier contexto académico, investigativo y laboral.

Es conveniente considerar que esta investigación tomó en cuenta el desarrollo en la UTNA de estudiantes que frecuentemente provienen de ámbitos académicos y sociales precarios, dado que la naturaleza de dicha institución es incorporar a la industria a jóvenes del ámbito rural, es decir, seguramente los resultados no reflejan la realidad que México anhela a partir del trabajo de instituciones de educación superior porque las bases de desempeño escolar son endebles en la población que conformó la muestra.

También es necesario advertir al lector que no se analizaron textos argumentativos con la profundidad que tal discurso implica, por ejemplo, el tipo de argumentos (generalizaciones, analogías, causas, deducciones), porque la actividad escolar de la UTNA no permite siquiera su generación, es decir, habría sido necesario y confortante para quienes firman este trabajo analizar el nivel de argumentación de los estudiantes si dicha forma discursiva fuese parte de la realidad concreta a la que se enfrentan y no solo de las buenas intenciones planteadas en los documentos base de la institución.

A partir de la investigación que aquí se describe, se sostiene que las diferentes formas discursivas deberían estar presentes en las estrategias de aprendizaje que realizan los estudiantes; es común que los educandos elaboren trabajos de tipo descriptivo, sin embargo, 


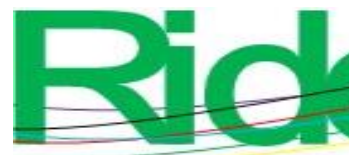

Revista Iberoamericana para la

Investigación y el Desarrollo Educativo

ISSN $2007-7467$

una formación superior de tinte técnico requiere imperiosamente un pronunciamiento participativo y crítico ante el avance tecnológico, ante su repercusión social y su finalidad ética, para ello es indispensable que la universidad tecnológica tome como reto formar a sus estudiantes en la argumentación.

\section{Referencias}

Bañales, G., Vega, N., Araujo, N., Reyna, A. y Rodríguez, B. (2015). La enseñanza de la argumentación escrita en la universidad. Una experiencia de intervención con estudiantes de lingüística aplicada. Revista Mexicana de Investigación Educativa, 20(66), 879-910. Recuperado de http://www.scielo.org.mx/pdf/rmie/v20n66/v20n66a9.pdf.

Carlino, P. (2013). Alfabetización académica diez años después. Revista Mexicana de Investigación Educativa, 18(57), 355-381. Recuperado de http://www.redalyc.org/articulo.oa?id=14025774003.

Cassany, D. (2012). Detalles sobre mi cocina. En Albarran, C. (comp.), Cómo escriben los que escriben. México: Fondo de Cultura Económica.

Coordinación General de Universidades Tecnológicas y Politécnicas [CGUT]. (2016). Coordinación académica. México: SEP-Coordinación General de Universidades Tecnológicas y politécnicas. Recuperado de http://cgut.sep.gob.mx.

Díaz, Á. (2015). La argumentación escrita. Didáctica y escritura. Medellín, Colombia: Universidad de Antioquia.

Fonseca, M. (2011). Comunicación oral y escrita. México: Pearson.

Gómez, J. (2012). La formación de estudiantes de grado undécimo en el campo de la escritura argumentativa. Estudio de caso de cuatro colegios públicos de Bogotá. Revista Iberoamericana sobre Calidad, Eficacia y Cambio en Educación, 10(2). Recuperado de: http://www.rinace.net/reice/numeros/arts/vol10num2/art7.pdf.

Lomas, C. (2017). El poder de las palabras. Enseñanza del lenguaje, educación democrática y ética de la comunicación. México: Santillana.

López, R. (2018). Condiciones del pensador crítico. Revista de la Universidad de La Salle, 1(76), 117-127. Recuperado de https://ciencia.lasalle.edu.co/ruls/vol2018/iss76/9/. 
Monzón, L. (2011). Argumentación: objeto olvidado para la investigación en México. Revista Electrónica de Investigación Educativa, 13(2), 41-54. Recuperado de http://redie.uabc.mx/vol13no2/contenido-monzon.html.

Morin, E. (2008). Introducción al pensamiento complejo. Barcelona, España: Gedisa.

Núñez, J. (2017). Los métodos mixtos en la investigación en educación: hacia un uso reflexivo. Cuadernos de Pesquisa, (47), 632-649. Recuperado de http://www.scielo.br/pdf/cp/v47n164/1980-5314-cp-47-164-00632.pdf.

Peña, L. (2008). La competencia oral y escrita en la educación superior. Documento realizado en el marco del Comité Consultivo para la Definición de Estándares y Evaluación de Competencias Básicas en la Educación Superior, conformado por iniciativa del Ministerio de Educación Nacional. Recuperado de WwW.mineducacion.gov.co/1621/articles-189357_archivo_pdf.

Pereira, Z. (2011). Los diseños de método mixto en la investigación en educación: Una experiencia concreta. Revista Electrónica Educare, 15(1), 15-29. Recuperado de https://www.redalyc.org/189357_archivo_pdf.

Pozo, J. (2012). Aprendices y maestros. La psicología cognitiva del aprendizaje. Madrid, España: Alianza.

Santibáñez, C. (2018). Origen y función de la argumentación. Pasos hacia una explicación evolutiva y cognitiva. Perú: Palestra Editores.

Weston, A. (2016). Las claves de la argumentación. México: Ariel.

Zambrano, J., Orozco, A. y Caro M. (2016). Estrategias para la enseñanza de la producción de textos argumentativos en el área de ciencias biológicas. Colombian Applied Linguistics Journal, 8(1), 43-55. 


\begin{tabular}{|c|c|}
\hline Rol de Contribución & Autor (es) \\
\hline Conceptualización & Mónica Muñoz Muñoz \\
\hline Metodología & Mónica Muñoz Muñoz \\
\hline Software & No Aplica \\
\hline Validación & $\begin{array}{l}\text { Mónica Muñoz Muñoz (Principal) / Yvonne Ibarra Peña } \\
\text { (Secundario) }\end{array}$ \\
\hline Análisis Formal & Yvonne Ibarra Peña \\
\hline Investigación & Yvonne Ibarra Peña \\
\hline Recursos & Yvonne Ibarra Peña \\
\hline Curación de datos & Yvonne Ibarra Peña \\
\hline $\begin{array}{l}\text { Escritura - Preparación del } \\
\text { borrador original }\end{array}$ & Mónica Muñoz Muñoz (Principal) Yvonne Ibarra (Secundario) \\
\hline $\begin{array}{l}\text { Escritura - Revisión y } \\
\text { edición }\end{array}$ & Mónica Muñoz Muñoz \\
\hline Visualización & Mónica Muñoz Muñoz \\
\hline Supervisión & Mónica Muñoz Muñoz \\
\hline $\begin{array}{l}\text { Administración de } \\
\text { Proyectos }\end{array}$ & Mónica Muñoz Muñoz \\
\hline Adquisición de fondos & Mónica Muñoz Muñoz \\
\hline
\end{tabular}

